\title{
MODULAÇÃO AUTONÔMICA E CAPACIDADE FUNCIONAL EM INDIVÍDUOS PORTADORES DE DIABETES MELLITUS DO TIPO 1 E 2
}

\section{AUTONOMIC MODULATION AND FUNCTIONAL CAPACITY IN DIABETES MELLITUS TYPE 1 AND 2 SUBJECTS}

\author{
Rafael Leite Alves ${ }^{1}$, Flávio Mariz Freitas ${ }^{2}$, \\ Alline Sancha Nascimento Fernandes ${ }^{2}$, Sabrina Campos Ferraz ${ }^{2}$, \\ Edson da Silva ${ }^{3}$, Clynton Lourenço Corrêa ${ }^{4}$, Luciana Duarte Novais Silva ${ }^{5}$
}

\section{RESUMO:}

Objetivos: avaliar a variabilidade da frequência cardíaca (VFC) durante testes autonômicos cardiovasculares e a capacidade funcional de indivíduos com DM tipos 1 e 2. Método: foram avaliados 15 indivíduos com DM e 12 indivíduos saudáveis, durante a realização de testes autonômicos cardiovasculares de manobra de acentuação da arritmia sinusal respiratória (ASR), handgrip e valsalva. Além disso, foi aplicado o teste submáximo de Paschoal para avaliação da capacidade funcional. Durante a realização dos testes autonômicos foi coletada a variabilidade da frequência cardíaca (VFC), por meio do registro dos intervalos RR, considerando-se os índices no domínio do tempo (RMSSD e pNN50) e da freqüência, como baixa e alta freqüência (BF e AF) e a relação entre os mesmos (BF/AF). Além da análise da VFC, foi registrado a distância percorrida durante o Teste de Paschoal e a glicemia capilar. Resultados: Os valores referentes à glicemia foram significativamente maiores $(p<0,05)$ no grupo DM1 e DM2 comparados aos controles. Os valores de pNN50 para o DM1, em todos os testes realizados, foram menores em relação ao CDM1. Nenhuma diferença estatística foi encontrada quando comparados DM2 com o CDM2. A distância percorrida no teste de Paschoal foi similar entre os grupos de indivíduos com DM e seus respectivos controles. Conclusão: A variável pNN50 foi menor nos indivíduos com DM1, sugerindo redução da atividade parassimpática nesses indivíduos, porém sem alteração da capacidade funcional, avaliada pelo teste de Paschoal quando comparados ao grupo controle.

Palavras-chave: diabetes mellitus; sistema nervoso autônomo; freqüência cardíaca; capacidade funcional.

1 Professor Substituto do Departamento de Ciências Básicas da Saúde da Universidade Federal dos Vales do Jequitinhonha e Mucuri (UFVJM), Rua da Glória, 187, Centro - CEP: 39100-000 - Diamantina (MG), Brasil

2 Fisioterapeutas graduados pela Universidade Federal dos Vales do Jequitinhonha e Mucuri (UFVJM) , Rua da Glória, 187, Centro - CEP: 39100-000 - Diamantina (MG), Brasil

3 Professor Assistente do Departamento de Ciências Básicas da Saúde da Universidade Federal dos Vales do Jequitinhonha e Mucuri (UFVJM), Rua da Glória, 187, Centro - CEP: 39100-000 - Diamantina (MG), Brasil

4 Professor Adjunto do Curso de Fisioterapia da Universidade Federal do Rio de Janeiro (UFRJ), Rua Prof Rodolpho Paulo Rocco, s/n, Ilha do Fundão - CEP: 21941-913 - Rio de Janeiro (RJ), Brasil

5 Professora Adjunta do Departamento de Fisioterapia Aplicada da Universidade Federal do Triângulo Mineiro, Avenida Getúlio Guaritá, 159, Bairro Abadia - CEP: 38025-440 - Uberaba (MG), Brasil

Trabalho Desenvolvido no Departamento de Fisioterapia da Universidade Federal dos Vales do Jequitinhonha e Mucuri com fomento da FAPEMIG/PIBIc Processo: APQ00595-08.

Correspondência para: lunovais@yahoo.com.br

Suggested citation: Alves, RL et al. Autonomic Modulation and Functional Capacity in Diabetes Mellitus Type 1 and 2 Subjects. J. Hum. Growth Dev. 2012; 22(3): 321-327

Manuscript submitted Feb 16 2012, accepted for publication Aug 102012. 


\begin{abstract}
:
Objectives: Evaluation of heart rate variability (HRV) during cardiovascular autonomic tests and functional capacity of patients with type 1 and type 2 DM. Methods: Fifteen individuals with DM and twelve healthy ones were evaluated during cardiovascular autonomic tests of maneuver enhancement of respiratory sinus arrhythmia (RSA), handgrip and Valsalva. In addition, the Paschoal test was applied to evaluate submaximal functional capacity. During the tests, autonomic heart rate variability (HRV) was collected by recording the RR intervals, considering the rates in the time domain (RMSSD and pNN50) and frequency domain, as low and high frequency (LF and HF) and the relationship between them (LF / HF). Besides the analysis of HRV and capillary glucose, the distance traveled during Paschoal test was also recorded. Results: The values related to blood glucose levels were significantly higher $(p<0.05)$ in DM1 and DM2 groups compared to controls. PNN50 values for DM1 in all tests were lower compared to CDM1. No statistical difference was found when compared DM2 with CDM2. The distance of Paschoal test was similar between groups of individuals with diabetes and their respective controls. Conclusion: The variable pNN50 was lower in individuals with DM1, suggesting reduced of the parasympathetic activity in these individuals, but no change in functional capacity, as measured by Paschoal test when compared to the control group.
\end{abstract}

Key words: diabetes mellitus, autonomic nervous system, heart rate, functional capacity.

\section{INTRODUÇÃO}

O diabetes Mellitus (DM) é um grupo das doenças metabólicas caracterizada pela hiperglicemia resultante dos defeitos na secreção ou ação da insulina, ou ambos ${ }^{1,2}$. A doença está associada ao aumento da mortalidade e ao alto risco de desenvolvimento de complicações micro e macrovasculares, bem como de neuropatias, cegueira, insuficiência renal e amputações de membros ${ }^{1,3}$. É responsável por gastos expressivos em saúde e substancial redução da capacidade de trabalho e da expectativa de vida ${ }^{1,2,3}$.

A neuropatia autonômica cardiovascular é uma forma comum de disfunção autonômica encontrada em pacientes com DM, provocando anormalidades no controle da freqüência cardíaca, e na dinâmica vascular ${ }^{4}$.

A disfunção autonômica pode ser avaliada por meio da variabilidade da frequência cardíaca (VFC), a qual reflete as oscilações periódicas da frequência cardíaca instantânea e da duração dos in- tervalos entre ondas $R$ ( $i R-R$ ) consecutivas do eletrocardiograma, estando portanto na dependência da atividade do sistema nervoso simpático e parassimpático $0^{5,6}$.

Para tal avaliação, alguns testes têm sido utilizados, desde os invasivos, por meio de bloqueios farmacológicos (bloqueio simples ou bloqueio duplo do simpático e do parassimpático cardía$\mathrm{co})^{7,8}$, até por manobras fisiológicas não invasivas, como manobra de Valsalva ${ }^{9}$, manobra postural ativa ${ }^{10,11}$ ou passiva ${ }^{12}$, realização de arritmia sinusal respiratória ${ }^{13}$, teste de imersão da face ou da mão em água gelada ${ }^{14}$ ou mudança de altitu$\mathrm{de}^{15}$. Entretanto, vale ressaltar que são raros os estudos que analisaram a VFC durante manobras autonômicas não invasivas em indivíduos com DM.

Por meio da avaliação da VFC durante manobras autonômicas é possível identificar objetivamente estágios iniciais da neuropatia autonômica cardiovascular e fornecer opções terapêuticas que se baseiam em controle dos sintomas e 
prevenção do avanço da doença. Sendo assim, é possível a prevenção de eventos cardíacos decorrentes de complicações da neuropatia autonômica, tais como taquicardia de repouso e infarto do miocárdio silencioso, entre outros ${ }^{16}$.

Outro aspecto importante para a compreensão da clínica do diabetes Mellitus é a investigação da capacidade funcional dos indivíduos acometidos. Foi publicado no Brasil, o teste de Paschoal que sugere a previsão da capacidade física a partir de um protocolo de esforço realizado em cicloergômetro, nos moldes do teste de caminhada de seis minutos $\left(\mathrm{TC}^{\prime}\right)^{17}$. Esse teste diferencia-se do TC6 ${ }^{\prime}$ por propor uma graduação da capacidade física a partir da distância atingida pelo indivíduo durante a realização do mesmo. Além disso, tem uma conotação biomecânica diferente, devido ao fato do indivíduo realizá-lo em posição sentada, o que pode revelar aspectos diferentes da capacidade funcional e das respostas cardíacas e respiratórias.

Assim, o objetivo é avaliar a variabilidade da frequência cardíaca (VFC) durante testes autonômicos cardiovasculares e a capacidade funcional em indivíduos com DM dos tipos 1 e 2.

\section{MÉTODO}

\section{Casuística}

No período compreendido entre setembro de 2008 e março de 2009, 145 pacientes com DM, cadastrados em Estratégias de Saúde da Família (ESF's) do município de Diamantina - MG foram triados e com base em seus prontuários e anotações do cadastro na unidade básica de saúde, 37 foram pré-selecionados para participar do presente estudo por obedecerem nesse momento aos critérios de inclusão.

Os indivíduos pré-selecionados foram submetidos a uma primeira avaliação, que consistia em anamnese e verificação da medicação em uso no momento. Desses 37 indivíduos, 15 aceitaram ou obedeciam aos critérios de inclusão, sendo os outros excluídos por estarem em uso de medicamentos beta- bloqueador ou bloqueador do canal de cálcio (16), apresentarem dificuldades para realização dos testes como osteoartrose comprometendo a marcha ou outras condições ortopédicas (1), condições psicológicas/neurológicas, como acidente vascular encefálico (2), ou pela incompatibilidade entre dias e horários das avaliações com a disponibilidade dos voluntários (3).

\section{Critérios de inclusão}

Os critérios de inclusão consistiam em: ser maior de dezoito anos ou quando menor, ter o termo de consentimento livre e esclarecido assinado pelos pais ou responsáveis; possuir diagnóstico de DM tipos 1 ou 2, com valores glicêmicos superiores a $126 \mathrm{mg} / \mathrm{dL}^{18}$.

\section{Critérios de exclusão}

Os critérios de exclusão adotados no presente estudo foram valores glicêmicos normais, hipertensão arterial em estágios 2 e $3^{19}$, diagnóstico de cardiopatias, pneumopatias ou instabilidade clínica, neuropatias não relacionadas à DM, a prática de atividade física regular há mais de 1 mês, uso de medicamentos betabloqueadores, bloqueadores de canal de cálcio e agonistas beta-adrenérgicos ou a inabilidade para compreender e realizar os testes.

\section{Aspectos éticos da pesquisa}

Os 27 indivíduos que concordaram em participar do estudo foram esclarecidos quanto aos procedimentos aos quais seriam submetidos e assinaram o termo de consentimento livre e esclarecido, aprovado pelo Comitê de Ética em Pesquisa da Universidade Federal dos Vales do Jequitinhonha e Mucuri sob o parecer número 008/09.

\section{Protocolo experimental}

Os voluntários foram divididos em dois grupos sendo eles: indivíduos com diagnóstico de DM do tipo 2 (DM2, $\mathrm{n}=10$ ) e indivíduos com diagnóstico de DM do tipo 1 (DM1, $\mathrm{n}=5$ ). Os grupos controle consistiram de indivíduos cadastrados nos ESF's ou membros da comu- 
nidade acadêmica convidados pelos pesquisadores, que também deveriam obedecer aos mesmos critérios de inclusão, à exceção do diagnóstico de DM 1 ou 2 . A amostra selecionada para o controle totalizou 12 indivíduos, distribuídos em dois grupos de acordo com a faixa etária que deveria ser similar ao grupo estudado: o grupo controle para Diabetes Mellitus 1 (CDM1, $\mathrm{n}=6$ ) e o grupo controle para Diabetes Mellitus 2 (CDM2, $n=6)$.

\section{Testes Autonômicos e Teste de Paschoal}

Os testes autonômicos cardiovasculares adotados foram a manobra de valsava, manobra de acentuação da Arritmia Sinusal Respiratória e Handgrip além do teste submáximo de Paschoal. Os mesmos foram realizados na seqüencia apresentada, com um intervalo de repouso para que as variáveis cardiovasculares retornassem a valores basais.

Os protocolos foram realizados com o indivíduo na posição sentada, sempre controlando a temperatura (entre $20^{\circ} \mathrm{C}$ e $25^{\circ} \mathrm{C}$ ) e umidade relativa do ar (entre 40 e $60 \%$ ).

Para cada protocolo realizado foi registrado um arquivo hrm no monitor de frequência cardíaca, contendo os períodos de repouso inicial e final e o período da manobra.

\section{Manobra de Valsalva ${ }^{12,20}$}

A manobra de Valsalva foi realizada com o indivíduo sentado, sendo esse orientado a manter a expiração forçada contra a glote fechada por 15 segundos com uma pressão expiratória de $40 \mathrm{mmHg}$ medida por manovacuômetro, respeitando-se dois minutos de repouso antes da realização da manobra e dois minutos posteriores à realização da mesma.

\section{Manobra de Acentuação da Arritmia Sinusal Respiratória ${ }^{13,20}$}

Durante a realização da manobra de acentuação da arritmia sinusal respiratória (ASR), com o voluntário sentado, o mesmo era orientado a inspirar e expirar profunda e lentamente, sendo tam- bém instruído a controlar a freqüência respiratória de acordo com o comando verbal do avaliador, permitindo-se 5 a 6 ciclos respiratórios por minuto numa coleta de aproximadamente 6 minutos, sendo os dois primeiros minutos de respiração espontânea, $3^{\circ}$ e $4^{\circ}$ minutos de ASR e o $5^{\circ}$ e $6^{\circ}$ minutos com retorno a respiração espontânea.

\section{Handgrip ${ }^{20}$}

Também conhecido como exercício estático, consistiu na medida da força de preensão, de três contrações máximas, escolhendo-se o maior valor entre as mesmas. Foi utilizado 30\% dessa intensidade em uma contração isométrica sustentada por, no mínimo, 2 minutos, com o indivíduo na posição sentada, respeitando-se os períodos de dois minutos de repouso antes e após a manobra. Tanto a medida da força, quanto a sua monitorização durante a contração isométrica sustentada, foi realizada com o auxílio de um dinamômetro.

\section{Teste de Paschoal ${ }^{17}$}

Por fim foi realizado o teste de Paschoal, utilizado em protocolos de avaliação da capacidade funcional cardiorrespiratória. Os voluntários foram instruídos a pedalar, em um cicloergômetro horizontal (Movement ${ }^{\circledR} \mathrm{H} 3$ ), na maior velocidade possível, por seis minutos sem resistência. A velocidade média mínima para validação foi de $20 \mathrm{Km} / \mathrm{h}$, e ao término do teste, a distância total percorrida foi registrada.

Entre cada manobra autonômica realizada foi intercalado um período de repouso para que a $\mathrm{FC}$ retornasse aos valores basais, porém foi considerado para análise da VFC apenas o período das manobras.

\section{Análise da VFC}

Em todos os protocolos de avaliação de modulação autonômica foi realizada a monitorização e registro dos dados de FC batimento a batimento cardíaco, por meio de um cardiofrequencímetro da marca Polar $\AA$ (modelo S810i, 2001, Finlândia). Tal equipamento está validado na literatura para ava- 
liação da modulação autonômica da freqüência cardíaca ${ }^{21} \mathrm{e}$, por ser de baixo custo é acessível à prática clínica.

A análise dos dados foi feita posteriormente à transferência desses para um computador utilizando-se uma interface infrared compatível com o modelo do cardiofrequêncímetro, utilizando o software Polar Precision Performance ${ }^{\circledR}$.

Após a transferência dos dados, os gráficos de FC eram analisados visualmente e caso fosse necessário era aplicado um filtro digital por meio do próprio software de análise. Além disso, foi observado se cada sinal coletado apresentava um mínimo de 256 pontos necessários para viabilizar a análise dos dados.

A VFC foi analisada considerandose índices no domínio do tempo, utilizando estatísticas simples e no domínio da frequência, que por meio da análise espectral permite decompor a VFC nos seus componentes oscilatórios fundamentais, definindo-os pela sua frequência e amplitude.

No domínio do tempo foram utilizados os índices:

- RMSSD: corresponde a raiz quadrada da somatória do quadrado das diferenças entre os iR-R no registro divididos pelo número de iR-R em um tempo determinado menos um iR-R 22 . É capaz de fornecer uma medida indireta específica da atividade parassimpática atuante no nó sino atrial ${ }^{23}$.

- pNN50: que corresponde a porcentagem em relação ao total de iR-R que tenham uma diferença superior a 50 milissegundos em relação aos $\mathrm{iR}-\mathrm{R}$ anteriores, refletindo também uma medida indireta do tônus parassimpático ${ }^{24}$. No domínio da frequência foram utilizados os índices:

- Baixa frequência (BF): com faixa de variação de 0,04 a $0,15 \mathrm{~Hz}$, que é decorrente da ação conjunta do componente vagal e simpático sobre o coração, sendo que o componente simpático é predominante. Portanto, essa banda de frequência tem sido utilizada como um marcador da modulação simpática atuante no coração ${ }^{5,6}$;

- Alta frequência (AF): com faixa de variação de 0,15 a $0,4 \mathrm{~Hz}$, que corresponde à modulação respiratória e é um indicador da atuação do vago sobre o coração ${ }^{5,6}$;

- Razão BF/AF: razão que reflete as interações absolutas e relativas entre os componentes simpáticos e parassimpáticos do sistema nervoso autônomo no coração $25,26$.

\section{Análise estatística}

Foi utilizado o Teste de KolmogorovSmirnov, constatando-se a distribuição normal das variáveis analisadas.

Foram comparados os dados de idade, características antropométricas, frequência cardíaca em repouso, pressão arterial sistólica e diastólica em repouso e glicemia entre os grupos de voluntários com diabetes e seus grupos controle, utilizando o teste $T$ não pareado.

Os índices obtidos por meio da aplicação dos protocolos experimentais (RMSSD, pNN50, BF, AF, BF/AF), foram comparados entre os grupos de indivíduos com DM e seus respectivos grupos controles, utilizando o teste $\mathrm{T}$ não pareado.

Todos os testes estatísticos utilizados neste estudo tiveram nível de significância estabelecido em 5\%.

\section{RESULTADO}

Os resultados do presente estudo são apresentados nas tabelas 1 e 2, em média e desvio padrão da média.

Observa-se na tabela 1 que os indivíduos dos grupos com DM apresentaram valores glicêmicos superiores $(p<0,05)$ quando comparados aos seus respectivos grupos controle. Além disso, a massa corporal e o índice de massa corporal (IMC) foram menores nos indivíduos do grupo DM1 quando comparados ao CDM1. 
Tabela 1: Caracterização dos Indivíduos estudados.

\begin{tabular}{|c|c|c|c|c|c|c|}
\hline & $\operatorname{DMI}(n=5)$ & $\operatorname{CDMI}(n=6)$ & $\mathbf{p}$ & $\operatorname{DMII}(n=10)$ & $\operatorname{CDMII}(n=6)$ & $\mathbf{p}$ \\
\hline Sexo & 1Masc./4Fem. & 3Masc./3Fem. & - & 2Masc./8Fem. & 3Masc./3Fem. & - \\
\hline Idade(anos) & $17.6 \pm 1.94$ & $21.17 \pm 3.19$ & 0.0577 & $56.9 \pm 10.59$ & $66.67 \pm 16.51$ & 0.1683 \\
\hline Massa Corporal (kg) & $56.52 \pm 5.76$ & $71.7 \pm 8.04$ & $0.0065 *$ & $73.86 \pm 13.93$ & $73.67 \pm 19.12$ & 0.9816 \\
\hline Estatura(metros) & $1.62 \pm 0.09$ & $1.72 \pm 0.07$ & 0.0715 & $1.57 \pm 0.12$ & $1.61 \pm 0.07$ & 0.4623 \\
\hline IMC $\left(\mathrm{kg} / \mathrm{m}^{2}\right)$ & $21.4 \pm 1.57$ & $24.01 \pm 1.19$ & $0.0121 *$ & $29.61 \pm 1.69$ & $28.13 \pm 5.71$ & 0.4481 \\
\hline PAS (mmHg) & $122 \pm 19.24$ & $102,67 \pm 10.63$ & 0.0633 & $131,2 \pm 20.55$ & $129,67 \pm 12.09$ & 0.8713 \\
\hline PAD (mmHg) & $78 \pm 10.95$ & $71,33 \pm 7.23$ & 0.2560 & $81,4 \pm 13.13$ & $77,67 \pm 4.27$ & 0.5155 \\
\hline FC rep.(bpm) & $95.2 \pm 23.56$ & $77.7 \pm 9.33$ & 0.1262 & $88.9 \pm 17.0$ & $77.67 \pm 11.62$ & 0.1769 \\
\hline Glicemia(mg/dL) & $429.2 \pm 79.69$ & $94.5 \pm 10.78$ & $<0.0001 *$ & $211.1 \pm 89.63$ & $101.67 \pm 15.80$ & $0.0111^{*}$ \\
\hline $\begin{array}{l}\text { Número de Indivíduos } \\
\text { Hipertensos }\end{array}$ & 0 & 0 & - & 4 & 1 & - \\
\hline
\end{tabular}

* $p<0.05$ - teste T não pareado

Fem. (sexo feminino); Masc.(sexo masculino) FC rep. (freqüência cardíaca em repouso) avaliada pelo pulso radial; IMC (índice de massa corporal); PAS (pressão arterial sistólica - $\mathrm{mmHg}$ ); PAD (pressão arterial diastólica - $\mathrm{mmHg}$ );

Na tabela 2, das variáveis consideradas durante os testes cardíacos autonômicos, a variável pNN50 foi menor no grupo DM1 comparado ao grupo CDM1 em todas as manobras realizadas. Além disso, a variável BF foi menor no grupo DM1 comparado ao CDM1 durante a ASR.
Não foram encontradas diferenças estatísticas nas comparações dos índices no domínio do tempo e da frequência quando comparados os grupos DM2 e CDM2.

Também não foram encontradas diferenças estatisticamente significantes na comparação entre as distâncias percorridas durante o teste de Paschoal.

Tabela 2. Variáveis RMSSD, pNN50, BF, AF, BF/AF durante testes autonômicos cardiovasculares e distância percorrida no teste de Paschoal em indivíduos com DM e seus respectivos controles.

\begin{tabular}{|c|c|c|c|c|c|c|c|}
\hline Manobras & Variáveis & $\operatorname{DMI}(n=5)$ & $\operatorname{CDMI}(n=6)$ & p & $\operatorname{DMII}(n=10)$ & $\operatorname{CDMII}(n=6)$ & p \\
\hline \multirow[t]{5}{*}{ ASR } & RMSSD & $69,86 \pm 41,27$ & $147,52 \pm 70,40$ & 0.0586 & $112,84 \pm 142,42$ & $113,35 \pm 68,53$ & 0.9936 \\
\hline & pNN50 & $4,2 \pm 5,78$ & $18,72 \pm 9,47$ & $0.0154 *$ & $4,72 \pm 5,56$ & $4,48 \pm 3,34$ & 0.9266 \\
\hline & BF & $1843,54 \pm 1711,65$ & $7749,37 \pm 4591,09$ & $0.0242 *$ & $9450,13 \pm 22953,84$ & $2460,63 \pm 1318,27$ & 0.4746 \\
\hline & $\mathrm{AF}$ & $1574,87 \pm 1594,83$ & $7287,50 \pm 5459,19$ & 0.0516 & $8411,20 \pm 21853,22$ & $4351,28 \pm 5455,78$ & 0.6659 \\
\hline & $\mathrm{BF} / \mathrm{AF}$ & $153,2 \pm 72,46$ & $145,07 \pm 76,39$ & 0.8612 & $216,09 \pm 181,48$ & $101,47 \pm 58,10$ & 0.1600 \\
\hline \multirow[t]{5}{*}{ Valsalva } & RMSSD & $108,54 \pm 60,33$ & $195,62 \pm 112,88$ & 0.1574 & $95,29 \pm 56,45$ & $136,52 \pm 92,05$ & 0.2813 \\
\hline & pNN50 & $5,94 \pm 7,25$ & $17,82 \pm 9,32$ & $0.0456 *$ & $2,36 \pm 3,09$ & $3,75 \pm 2,83$ & 0.3842 \\
\hline & BF & $2589,58 \pm 2629,90$ & $11275,78 \pm 8376,80$ & 0.0543 & $3025,91 \pm 2511,51$ & $4487,23 \pm 5029,52$ & 0.4471 \\
\hline & $\mathrm{AF}$ & $3638,85 \pm 4442,97$ & $14089,61 \pm 13012,15$ & 0.1230 & $3323,90 \pm 3959,08$ & $6134,53 \pm 8376,75$ & 0.3741 \\
\hline & $\mathrm{BF} / \mathrm{AF}$ & $68,8 \pm 18,57$ & $104,8 \pm 46,90$ & 0.1434 & $153,54 \pm 140,87$ & $130,57 \pm 132,54$ & 0.7519 \\
\hline \multirow[t]{5}{*}{ Handgrip } & RMSSD & $65,86 \pm 46,12$ & $78,53 \pm 38,09$ & 0.6290 & $109,23 \pm 51,53$ & $139,92 \pm 81,83$ & 0.3690 \\
\hline & pNN50 & $2,08 \pm 1,82$ & $9,78 \pm 4,94$ & $0.0095 *$ & $2,25 \pm 2,41$ & $2,55 \pm 2,81$ & 0.8240 \\
\hline & $\mathrm{BF}$ & $2584,07 \pm 1799,32$ & $3432,93 \pm 1601,58$ & 0.4289 & $2173,19 \pm 1691,87$ & $8711,84 \pm 14531,25$ & 0.1717 \\
\hline & $\mathrm{AF}$ & $1893,31 \pm 2825,76$ & $2256,68 \pm 2479,30$ & 0.8252 & $3687,09 \pm 3361,50$ & $5757,14 \pm 5630,91$ & 0.3682 \\
\hline & $\mathrm{BF} / \mathrm{AF}$ & $253,44 \pm 183,83$ & $271,92 \pm 276,44$ & 0.9015 & $66,91 \pm 20,14$ & $141,27 \pm 154,25$ & 0.1462 \\
\hline Paschoal & Distância $\quad(\mathrm{Km})$ & $3,08 \pm 0,54$ & $3,17 \pm 0,34$ & 0.7406 & $2,65 \pm 0,43$ & $2,60 \pm 0,58$ & 0.8681 \\
\hline
\end{tabular}

* $\mathrm{p}<0,05$ - teste t não pareado; ASR: arritmia sinusal respiratória. 


\section{DISCUSSÃO}

No presente trabalho, utilizamos a VFC para avaliar a modulação autonômica da frequência cardíaca, por se tratar de um método não-invasivo, eficaz e de baixo custo.

Estudos relatam importantes alterações da VFC em função da idade, do nível de condicionamento físico ${ }^{27}$ e da presença de fatores de risco para doença da artéria coronária (DAC ${ }^{28}$ ou DAC estabelecida29.

Além desses fatores, a literatura também mostra que frequência cardíaca $(F C)$ e a pressão arterial sistólica (PAS) e diastólica (PAD) de repouso são influenciadas por: características genéticas, características antropométricas (massa corporal e altura), sexo, fatores hormonais e emocionais, posição corporal, influências ambientais, nível de aptidão física, presença de doenças, entre outros $^{9}$.

No presente estudo, os valores referentes à massa corporal e ao IMC foram significativamente menores no DM1 quando comparados ao CDM1. Entretanto, para ambos os grupos os valores se encontravam dentro da faixa de normalidade ${ }^{18}$. A FC, a PAS e a PAD em repouso não apresentaram diferença significativa entre os grupos estudados. Como esperado, os valores glicêmicos nos grupos com diagnóstico de Diabetes Mellitus foi superior quando comparado aos seus respectivos controles, o que comprova a presença de um distúrbio primário, seja na liberação ou captação da insulina ou em ambos os mecanismos levando aos altos valores glicêmicos encontrados.

Em relação aos índices no domínio do tempo, os valores do índice RMSSD, não apresentaram diferenças estatisticamente significantes nas comparações entre os grupos com DM e seus respectivos controles. Entretanto, o grupo DM1 apresentou valores significativamente menores da variável pNN50 quando comparado ao grupo controle CDM1 em todas as manobras autonômicas realizadas, sugerindo assim, menor atividade vagal no grupo DM1.
De acordo com os dados da variável pNN50, os resultados do presente estudo estão de acordo com estudos prévios que encontraram redução da atividade vagal em indivíduos com diagnóstico de $\mathrm{DM} 1^{30,31}$.

Considerando os valores de RMSSD obtidos no presente trabalho, são observados valores médios menores nos indivíduos com DM1 apesar de não terem atingido significância estatística. Porém pode-se notar que há grande dispersão dos dados, observado pelo alto desvio padrão em todas as manobras utilizadas, podendo justificar a ausência de diferença estatística.

Em relação aos resultados obtidos no domínio da frequência, foram encontrados valores significativamente maiores da banda BF para os indivíduos do grupo CDM1 durante a manobra de acentuação da arritmia sinusal respiratória. Porém, estes indivíduos também apresentaram tendência a maiores valores da banda $A F$, mesmo não apresentando significância estatística, fazendo com que não houvesse diferença significativa na razão $\mathrm{BF} / \mathrm{AF}$, mas essa com tendência a menores valores no CDM1.

Alguns estudos têm demonstrado que uma forma melhor de caracterizar o balanço simpato-vagal é a utilização da razão $B F / A F$, que reflete as interações absolutas e relativas entre os componentes simpático e parassimpático do sistema nervoso autônomo no coração ${ }^{5}$.

Os resultados referentes ao domínio da frequência, no grupo DM1, são discordantes de outros estudos prévios que encontraram diferença estatisticamente significativa, mostrando uma neuropatia autonômica diabética em fase precoce do surgimento da doença $28,31,32$.

A possível justificativa para a ausência de diferenças significantes no presente trabalho é o tempo de diagnóstico da doença. Como não foi padronizado o tempo de diagnóstico de DM1 para inclusão dos indivíduos no estudo, esse dado foi heterogêneo, podendo ser o motivo para grande dispersão dos dados e ausência de diferença significativa.

Em relação aos indivíduos com DM2 não foram encontradas diferenças esta- 
tisticamente significantes em nenhum dos índices avaliados, durante manobras autonômicas.

Este resultado se assemelha aos resultados de estudos prévios que avaliaram a VFC durante manobras autonômicas. Em um estudo recente da VFC, foram utilizados testes como a manobra de Valsalva e respiração profunda, não se encontrando diferenças estatisticamente significantes entre indivíduos saudáveis e aqueles com diagnóstico precoce do diabetes ${ }^{33}$. Outro estudo em 2008, avaliou mulheres saudáveis e obesas com e sem DM2 durante manobra de Handgrip, não encontrando diferenças estatisticamente significantes entre os grupos $^{34}$.

Os resultados do presente estudo discordam de outros encontrados na literatura, onde indivíduos com DM2 apresentam maior tônus simpático em comparação a indivíduos saudáveis 35,36,37.

Os valores de distância percorrida durante a realização do Teste de Paschoal não apresentaram diferença estatisticamente significante. Esse teste propõe uma graduação da capacidade física a partir da distância percorrida em bicicleta ergométrica ${ }^{17}$, em intensidade submáxima (níveis de atividade de vida diária).

De acordo com a literatura é esperado menor capacidade aeróbia em indivíduos diagnosticados com Diabetes Mellitus ${ }^{38,39}$. Uma possível explicação para os resultados do presente estudo em relação à capacidade funcional, é que o protocolo de avaliação aplicado, utiliza intensidade submáxima de esforço. Sendo assim, é possível que a limitação

\section{REFERÊNCIAS}

1. Davidson $M B$, Defrozon RA, Drash $A$, Raskin P. Report of the Expert Committee on the Diagnosis and Classification of Diabetes Mellitus. Diabetes Care. 2003;26:5-20.

2. Torres HC, Hortale VA, Schall V. A experiência de jogos em grupos operativos na educação em saúde para di- funcional decorrente do DM ocorra em níveis de exercícios físicos mais altos, não limitando as atividades de vida diária dos indivíduos com DM.

O tamanho da amostra bem como a falta do registro referente ao tempo da doença podem ser apontadas como fatores limitantes desse estudo. Ainda deve ser considerada a dificuldade para seleção de uma amostra que preencha integralmente os critérios de inclusão, tendo em vista a alta porcentagem de voluntários com DM com associação a outras doenças, como a hipertensão arterial sistêmica e o uso concomitante de fármacos como os bloqueadores do canal de cálcio ou beta-bloqueadores, que alteram sobremaneira a modulação autonômica.

Assim, a variável pNN50 foi significativamente menor nos indivíduos com Diabetes Mellitus do tipo $1 \mathrm{em}$ todos os testes autonômicos cardiovasculares realizados, demonstrando redução da atividade parassimpática nesses indivíduos quando comparado ao grupo controle. Já, a capacidade funcional avaliada em níveis submáximos foi similar entre os indivíduos com e sem Diabetes Mellitus, inferindo-se dessa forma que a limitação às atividades em função da alteração da VFC encontrada, ocorra em níveis mais elevados de exercício físico na amostra considerada.

\section{Agradecimentos}

À Fundação de Amparo à Pesquisa de Minas Gerais - FAPEMIG/PIBIC pelo apoio financeiro ao projeto (Processo APQ00595-08).

abéticos. Cad. Saúde Pública. 2003;19(4):1039-1047.

3. Ortiz MCA, Zanetti ML. Levantamento dos fatores de risco para diabetes mellitus tipo 2 em uma instituição de ensino superior. Rev Latino-am Enfermagem. 2001;9(3):58-63.

4. Maser RE, Lenhard MJ. Cardiovascular Autonomic Neuropathy Due to Diabetes Mellitus: Clinical Manifes- 
tations, Consequences, and Treatment. J Clin Endocrinol Metab. 2005; 90(10):5896-5903.

5. Task Force of European Society of Cardiology of the North American Society of Pacing Electrophysiology. Heart rate variability. Standars of mensurement, physiological interpretation and clinical use. Circulation. 1996;93:1043-65.

6. Longo A, Ferreira D, Correia MJ. Variabilidade da Freqüência Cardíaca. Rev Port Cardiol.1995;14(3):241-262.

7. Akselrod S, Gordon D, Ubel FA, Shannon DC, Berger AC, Cohen RJ. Power spectrum analysis of heart rate fluctuaction: a quantitative probe of beat-to-beat cardiovascular control. Science. 1981;213:220-222.

8. Pomeranz B, Macauley RJ, Caudill MA, Kutz I, Adam D, Gordon D. Assessment of autonomic function in humans by heart rate spectral analysis. Am J Physiol. 1985;248: H151-H153.

9. Marães VRFS, Santos MDB, Catai AM, Moraes FR, Oliveira L, Gallo Jr L, et al. Modulação do sistema nervoso autonômico na resposta da freqüência cardíaca em repouso e à manobra de valsalva com o incremento da idade. Rev Bras Fisioter. 2004; 8(2): 97-103.

10. Marães VRFS. Estudo da variabilidade da freqüência cardíaca durante exercício físico dinâmico em voluntários sadios [dissertação de mestrado]. Campinas: Universidade Estadual de Campinas. Instituto de Biologia, 1999.

11. Ribeiro TF, Azevedo GD, Crescêncio JC, Marães VRFS, Papa V, Catai AM. Heart rate variability under resting conditions in postmenopausal and young women. Braz J Med Biol Res. 2001;34(7):871-7.

12. Martinelli FS. Resposta da freqüência cardíaca e da pressão arterial sistêmica às manobras posturais passivas e de Valsalva, em indivíduos sedentários e atletas corredores de longa distância [dissertação de mestrado]. Campinas: Universidade Estadual de Campinas, 1996.
13. Santos MDB, Moraes FR, Marães VRFS, Sakabe DI, Takahashi ACM, Oliveira L, et al. Estudo da arritmia sinusal respiratória e da variabilidade da freqüência cardíaca de homens jovens e de meia idade. Rev. Soc. Cardiol. 2003;13(3):15-26.

14. Perini R, Milesi S, Biancardi L, Pendergast DR, Veicsteinas $A$. Heart rate variability in exercising humans: effect of water immersion. Eur Appl Physiol 1998;77:326-332.

15. Perini R, Milesi $S$, Biancardi L, Veicsteinas A. Effects of high altitude acclimatization on heart rate variability in resting humans. Eur $\mathrm{J} \mathrm{Appl}$ Physiol. 1996;73: 521-528.

16. Vinik AI, Ziegler D. Diabetic Cardiovascular Autonomic Neuropathy. Circulation. 2007;115:387-397.

17. Paschoal MA. Avaliação cardiorrespiratória de esforço e programa de recondicionamento aeróbio em cicloergômetro para pacientes com doença pulmonar obstrutiva crônica. Rev Bras Fisioter. 2002; 6(3):119-25.

18. Sociedade Brasileira de Diabetes. Atualização Brasileira sobre Diabetes, 2005.

19. V Diretrizes Brasileiras de Hipertensão Arterial. Arq. Bras. Cardiol. São Paulo Sept. 2007; 89(3).

20. Castro CLB, Nóbrega ACL, Araújo CGS. Testes Autonômicos Cardiovasculares. Uma Revisão Crítica. Parte I e II. Arq Bras Cardiol. 1992;59:7583/151-158.

21. Vanderlei LCM, et al. Comparison of the Polar S810i monitor and the ECG for the analysis of heart rate variability in the time and frequency domains. Braz J Med Biol Res. 2008; 41(10):854-859.

22. Antila K. Quantitativa characterization of heart rate during exercise. Scand J Clin Lab Invest. 1979; 80:153-155.

23. Kleiger RE, Stein KP, Bosner MS, Rottman JN. The time-domain mensurements of heart variability. Armonk, Futura. 1995: 33-46.

24. Pumprla J, Howorka K, Groves D, Chester M, Nolan J. Functional Assessment of Heart Rate Variability: 
Physiological Basis and Practical Applications. Int. J. Cardiol. 2002; 84:1-14.

25. Lombardi F, Malliani A, Pagani M, Cerutti $S$. Heart rate variability and its sympatho-vagal modulation. Ospedale L. Sacco, Università di Milano, Italy. 1: Cardiovasc Res. 1996; 32(2): 208-216.

26. Pagani $M$, Lucini $D$, Pizzinelli $P$, Sergi M, Bosisio E, Mela GS, et al. Effects of aging and of chronic obstructive pulmonary disease on RR interval variability. J Auton Nerv Syst. 1996; 59:125-132.

27. Melo RC, et al. Effects of age and physical activity on the autonomic control of heart rate in healthy men. Braz J Med Biol Res. 2005;38(9):1331-38.

28. Pavy-Le Traon A, Fontaine S, Tap G, Guidolin B, Senard JM, Hanaire H. Cardiovascular autonomic neuropathy and other complications in type 1 diabetes. Clin Auton Res. 2010; 20(3): 153-60.

29. Günther A, Witte OW, Hoyer D. Autonomic dysfunction and risk stratification assessed from heart rate pattern. Open Neurol J. 2010; 15(4): 39-49.

30. Evans JM, Ziegler MG, Patwardhan AR, Ott JB, Kim CS, Leonelli FM, et al. Gender differences in autonomic cardiovascular regulation: spectral, hormonal, and hemodynamic indexes. J Appl Physiol. 2001; 91(6): 2611-8.

31. Waden J, Forsblom C, Thorn LM, Saraheimo $M$, Rosengård-Bärlund $M$, Heikkilä $O$, et al. Adult stature and diabetes complications in patients with type 1 diabetes: the FinnDiane study and the diabetes control and complications trial. Diabetes. 2009;58:1914-1920.

32. Boysen A, Lewin MAG, Hecker W, Leichter $\mathrm{HE}$, Uhlemann F. Autonomic function testing in children and adolescents with diabetes mellitus. Pediatric Diabetes. 2007; 8: 261-264.

33. Cabezas-Cerrato J, Hermida RC, Cabezas-Agrícola JM, Ayala DE. Cardiac Autonomic Neuropathy, Estimated Cardiovascular Risk, and Circadian Blood Pressure Pattern in Diabetes Mellitus. Chronobiol Int. 2009; 26(5):942-957.

34. Franklin RM, Baynard T, Weinstock RS, Goulopoulou S, Carhart Jr R, Ploutz-Snyder R, Figueroa A, Fernhall $B$, Kanaley JA. Autonomic responses to physiological stressors in women with type 2 diabetes. Clin Auton Res. 2008; 18: 66-73

35. Barbosa FJ, Barbosa PRB, Cordovil I. Modulação autonômica do coração na Hipertensão Arterial Sistêmica. Arq Bras Cardiol. 2002;78(2):181-188.

36. Balcioglu S, Ugur A, Turkoglu S, Ozdemir M, Çengel A. Heart Rate variability and Heart Rate Turbulence in Patients With Type 2 Diabetes Mellitus With Versus Without Cardiac Autonomic Neuropathy. Am J Cardiol. 2007; 100:890-893.

37. Masi CM, Hawkley LC, Rickett EM, Cacioppo JT. Respiratory sinus arrhythmia and diseases of aging: Obesity, diabetes mellitus, and hypertension. Biological Psychology. 2007; 74: 212-223.

38. Gregoire J, Tuck S, Yamamoto Y, Hughson RL. Heart rate variability at rest and exercise: influence of age, gender, and physical training. Can J Appl Physiol. 1996;21(6):455-470.

39. Lyerly GW, Sui X, Lavie CJ, Church TS, Hand GA, Blair SN. The association between cardiorespiratory fitness and risk of all-cause mortality among women with impaired fasting glucose or undiagnosed diabetes mellitus. Mayo Clin Proc. 2009; 84(9): 776-779. 